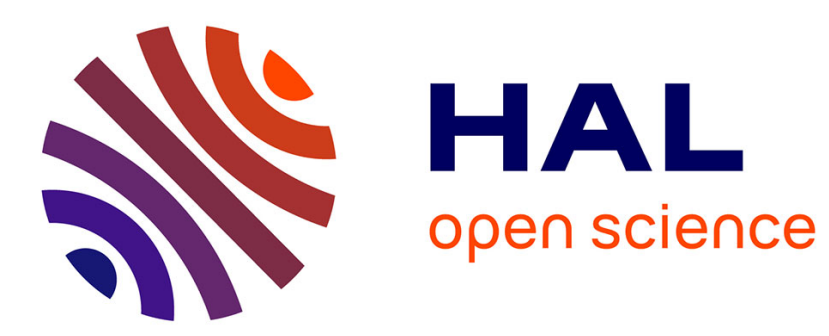

\title{
Comment l'éclat d'Homère se reflète chez Sappho
}

\author{
Nadine Le Meur-Weissman
}

\section{To cite this version:}

Nadine Le Meur-Weissman. Comment l'éclat d'Homère se reflète chez Sappho. Gaïa - Revue interdisciplinaire sur la Grèce archaïque, 2006, 10 (1), pp.189-201. 10.3406/gaia.2006.1498 . hal-02304264

\section{HAL Id: hal-02304264 \\ https://hal.science/hal-02304264}

Submitted on 3 Oct 2019

HAL is a multi-disciplinary open access archive for the deposit and dissemination of scientific research documents, whether they are published or not. The documents may come from teaching and research institutions in France or abroad, or from public or private research centers.
L'archive ouverte pluridisciplinaire HAL, est destinée au dépôt et à la diffusion de documents scientifiques de niveau recherche, publiés ou non, émanant des établissements d'enseignement et de recherche français ou étrangers, des laboratoires publics ou privés. 


\section{Comment l'éclat d'Homère se reflète chez Sappho}

\section{Nadine Le Meur-Weissman}

\section{Abstract}

How the Homeric notion of brilliance reflects in Sappho's poetry

The Homeric notion of brilliance, which underlines the male vertues of courage and fierceness and applies to the best warriors, is surprisingly also found in Sappho's poetry. The poetess particularly uses this image in her love poems to enhance the beauty of a woman or the destructive power of love. Sappho plays and rivals with the epic model by proposing a new interpretation of a well-known motif.

\section{Résumé}

La notion homérique d'éclat, qui souligne l'excellence toute masculine du guerrier dans l'épopée, tient aussi, paradoxalement, une place importante dans la poésie de Sappho. La poétesse utilise ce thème en particulier dans les poèmes évoquant l'amour, pour mettre en valeur la beauté d'une femme ou encore la puissance destructrice du sentiment amoureux. Elle joue ainsi clairement avec le modèle homérique et rivalise avec le poète épique, en proposant une nouvelle interprétation d'un motif bien connu.

Citer ce document / Cite this document :

Le Meur-Weissman Nadine. Comment l'éclat d'Homère se reflète chez Sappho. In: Gaia : revue interdisciplinaire sur la Grèce Archaïque, numéro 10, 2006. pp. 189-201;

doi : https://doi.org/10.3406/gaia.2006.1498

https://www.persee.fr/doc/gaia_1287-3349_2006_num_10_1_1498

Fichier pdf généré le 17/01/2019 


\title{
Comment l'éclat d'Homère se reflète chez Sappho
}

\author{
NADINE LE MEUR-WeISSMAN \\ École normale supérieure en Lettres et Sciences humaines (ENS Lyon)
}

Le monde d'Homère met en scène des héros guerriers, aux exploits sur-humains et dont la valeur se révèle en particulier lors de batailles opposant des foules en armes. De même que le poète garde l'anonymat, le récit homérique s'intéresse peu à l'intériorité psychologique de ses personnages et reste relativement discret sur les sentiments que ceux-ci peuvent éprouver. La poésie lyrique au contraire n'hésite pas à nommer son auteur - qui dit «je $»-$, et déplace le centre d'intérêt vers la sphère du privé, de l'intime, de l'émotion, comme en témoigne pleinement la poésie de Sappho. Pourtant, l'opposition entre les deux mondes, ou les deux poètes, n'est peut-être pas toujours aussi nette et il est frappant de voir que certains thèmes ou certains motifs se retrouvent chez l'un comme chez l'autre. C'est le cas notamment de l'un des motifs les plus fréquents de la poésie homérique, celui de l'éclat, qui occupe également dans l'œuvre de Sappho une place récurrente. Chez Homère, l'une des fonctions principales de cet éclat est de souligner l'excellence et la supériorité d'un guerrier, et en particulier celle de son armement, c'est-à-dire ce qui, de l'extérieur, le distingue des autres soldats sur le champ de bataille. L'intérêt de ce motif, souvent développé et mis en valeur par une comparaison, réside en grande partie dans l'effet qu'il a, comme spectacle, sur ceux qui le voient. Il engendre souvent la frayeur, car il véhicule, comme le feu auquel il est lié, un symbole inquiétant et destructeur. Ainsi, au chant XXII de l'Iliade (v. 134-136), le poètc décrit de la manière suivante l'apparition d'Achille sur le champ de bataille : 


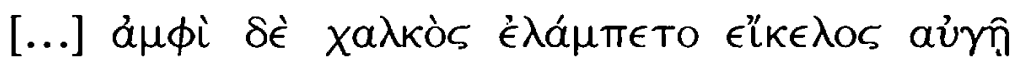

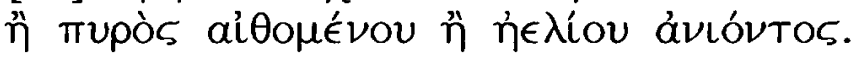

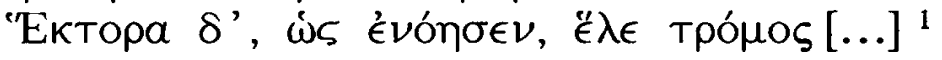

[...] et tout autour, le bronze resplendissait, semblable à l'éclat

du feu flamboyant ou du soleil levant.

Hector, dès qu'il l'aperçut, fut pris de terreur [...].

Il peut donc sembler étonnant de retrouver un tel motif dans la poésie toute personnelle et intimiste de Sappho. Aussi allons-nous essayer de montrer ici sous quelle forme se révèle sa présence, quelle est la pertinence du thème de l'éclat dans ces textes, et surtout de déterminer quelle relation la poétesse entretient avec la tradition homérique à travers lui : comment elle l'imite, la continue et la prolonge, rivalise avec elle ou éventuellement aussi s'en détache et pour quelles raisons.

Le poème 16 de Sappho, qui débute par la célèbre priamel, présente une première occurrence de la notion d'éclat avec le substantif

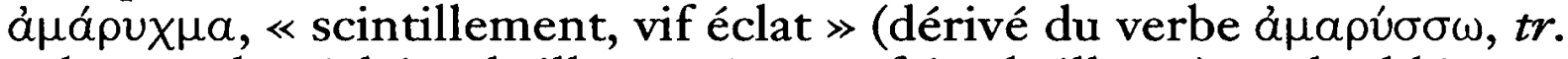
« lancer des éclairs, briller »; intr. « faire briller »), redoublée par l'adjectif $\lambda \alpha ́ \mu \pi \rho \circ \varsigma, \ll$ brillant » (v. 17-18):

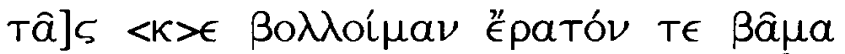

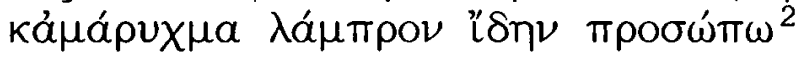

(Anactoria) dont j'aimerais voir la démarche désirable et le rayonnant éclat du visage ${ }^{3}$.

La « démarche désirable » et le « rayonnant éclat du visage » sont les seuls éléments de description que nous donne Sappho du personnage d'Anactoria. On remarque d'emblée le fort lien institué par la coordination $\tau \epsilon$. kaí entre $\beta a ̂ \mu \alpha$ et ả $\mu \alpha ́ \rho v \chi \mu \alpha$, en tant que deux manifestations de la beauté de la jeune femme: son caractère désirable est ainsi représenté à la fois par le mouvement et par l'éclat ${ }^{4}$.

1. Tous les textes de l'lliade sont cités d'après l'édition de Paul Mazon, Paris, Belles Lettres, CUF, 1987 (1 ${ }^{\text {rc }}$ édition, 1937).

2. Les textes de Sappho sont cités d'après l'édition d'E.M. Voigt, Sappho et Alcaeus, Amsterdam, Athenaeum - Polack \& van Gennep, 1971.

3. On trouve également dans un poème malheureusement très fragmentaire (poème 4, v. 6-7) une autre association du visage et de la notion d'éclat :

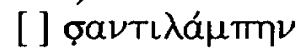

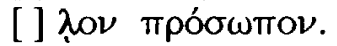

réfléchir la lumière

(beau?) visage

4. Voir D. E. Gerber, A Companion to the Greek Lyric Poets, p. 173-175. 
L'emploi du mot rare ả $\mu \alpha ́ \rho v \gamma \mu \alpha$ peut s'expliquer de diverses manières : il n'est peut-être dû qu'à une simple volonté de Sappho de varier, d'innover par rapport à Homère, chez qui le substantif

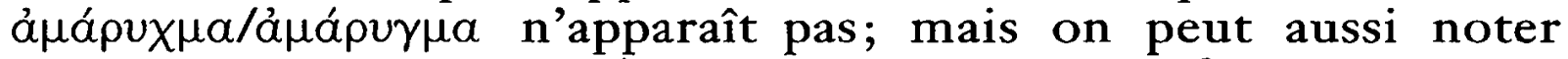
qu'ả $\mu a ́ p v \gamma \mu \alpha$ a un second sens, « mouvement vif et gracieux », qu'utilise Hésiode à propos des Charites (fragment 21 , v. 94) ${ }^{5}$ : c'est peut-être aussi l'existence de ce double sens qui a motivé le choix par l'auteur de ce substantif rare. D'autre part, les deux adjectifs $\lambda a ́ \mu \pi \rho \circ \nu$

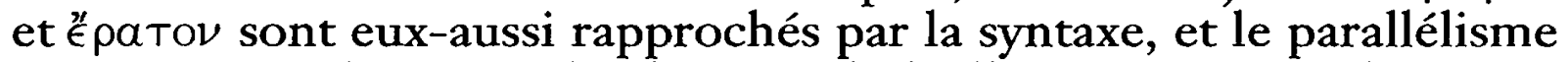
est encore souligné par le chiasme: le brillant est mis sur le même plan que le désirable et entre ainsi dans la sphère de l'amour ${ }^{6},-$ quittant celle de la guerre ${ }^{7}$.

Enfin, en qualifiant le visage de la jeune femme de $\lambda a ́ \mu \pi \rho o s$, Sappho investit le personnage de la qualité précise qui distingue les guer-

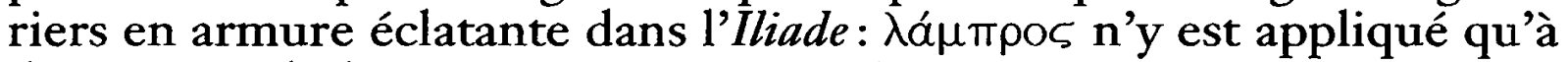
des parties de l'équipement guerrier (cuirasse, casque, cimier) ou à des corps célestes (soleil, astres). Et on trouve même, à plusieurs reprises, convergence de ces deux applications, comme c'est le cas au chant XXII (v. 25-32), quand Achille, approchant Troie dans son armure divine, est comparé à Sirius (《le Chien d'Orion $\gg)^{8}$ :

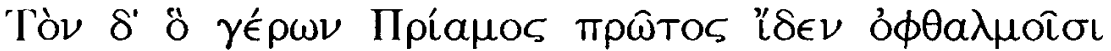

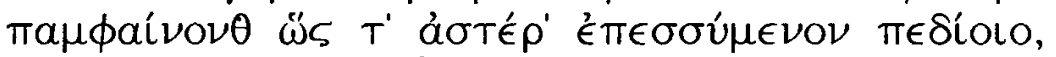

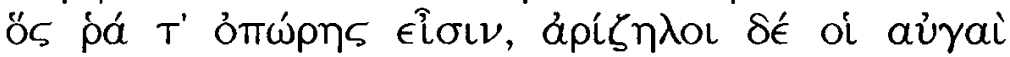

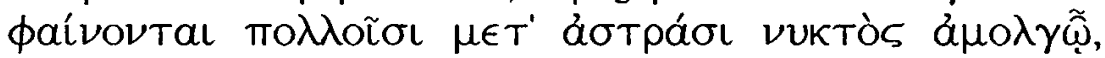

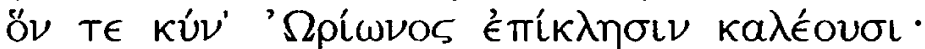

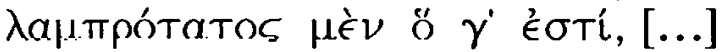

5. Voir D. L. Page, Sappho and Alcaeus, p. 54.

6. On peut également mentionner à cet égard le v. 26 du poème 58 de Sappho : Tò

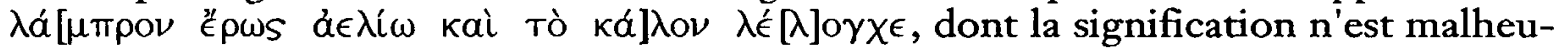
reusement pas assurée, étant donné l'état très lacunaire du texte, - Athénée en propose la paraphrase suivante : «l'amour du soleil (c'est-à-dire l'amour de la vie) a en lui pour moi à la fois le brillant et le bon». Si le sens de ce vers ne se laisse pas facilement appréhender, il est du moins possible de relever l'association, intéressante et nouvelle, faite par Sappho de «l'amour», du «brillant» et du «beau».

7. Voir L. Rissman, Love as war, p. 30 ss.

8. De même, au chant $V$ (v. 4-7), Athéna intervient afin que Diomède se distingue entre les Argiens et le poète illustre l'éclat particulier qui émane du guerrier à l'aide également de la comparaison avec Sirius :

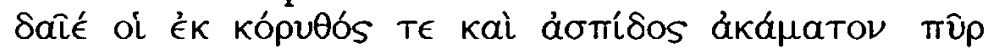

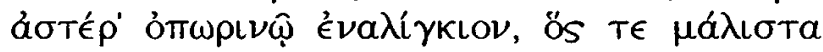

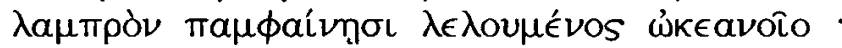

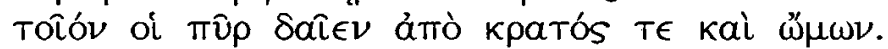

Sur son casque et son bouclier elle alluma un feu vicace.

On aurait dit l'astre de l'arrière-saison, qui resplendit

d'un éclat sans rival, quand il sort de son bain dans les eaux d'Océan.

Tout pareil était le feu que Pallas lui alluma sur la tête et sur les épaules. 
C'est le vieux Priam, le premier, qui de ses yeux l'aperçut, bondissant dans la plaine, resplendissant comme l'astre qui vient à l'arrière-saison et dont les feux éblouissants éclatent au milieu des étoiles sans nombre, au plein cœur de la nuit. On l'appelle le Chien d'Orion, et son éclat est sans pareil, [...].

L'emploi que fait Sappho de $\lambda a ́ \mu \pi \rho \circ s$, dans le poème 16, pour qualifier le visage de la jeune fille n'est pas le seul lien que celle-ci entretienne avec le monde de la guerre. Les vers 19-20 de ce même poème établissent en effet une comparaison explicite, et pour le moins surprenante, de la jeune fille avec des éléments insolites dans ce contexte:

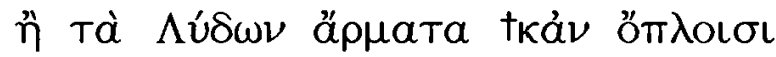

$\pi \epsilon \sigma \delta \mathrm{o \mu}] a ́ x \in \nu T a \zeta$.

les chars de Lydie et

les fantassins en armes.

Il peut tout d'abord sembler étrange qu'on puisse même songer à comparer les deux, c'est-à-dire à envisager comme possible d'aimer des chars et des fantassins. Mais le fait n'est pas sans exemple chez Homère précisément, où l'on trouve comme objet du verbe é $\rho a \mu a l$ :

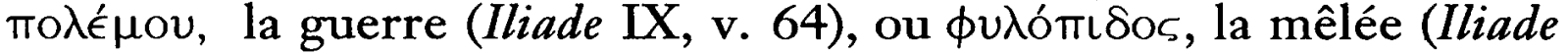
XVI, v. 208). Cette comparaison prend cependant son sens dans le poème si l'on se souvient de la priamel qui le débute :

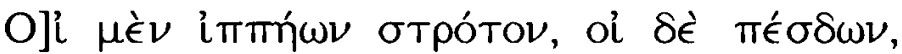

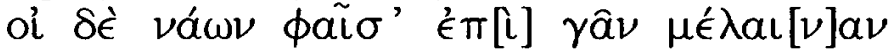

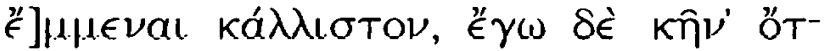

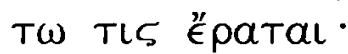

Les uns disent qu'une troupe de cavaliers, les autres de fantassins, les autres une flotte de navires, est ce qu'il y a de plus beau sur la terre noire; moi, je dis

que c'est l'être que l'on aime.

Outre le rappel du verbe ëpataı (v. 4) dans l'adjectif ë $\rho a \tau o \nu$ (v. 17), qui illustre le thème de l'amour, on trouve aussi déjà, dans ce préambule, la mention d'éléments appartenant à l'arsenal guerrier; la construction cyclique ${ }^{9}$ de cette partie du poème a d'ailleurs fait penser que celui-ci prenait fin au vers 20 . Il est donc clair que les deux thèmes de l'amour et de la guerre s'entremêlent dans ce poème et s'y confrontent même, puisque, aux deux reprises mentionnées, la poétesse marque ostensiblement sa préférence pour l'amour: on peut

9. Voir F. Lasserre, Sappbo, une autre lecture, p. 161 ss. 


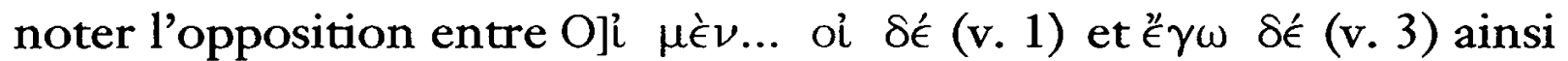

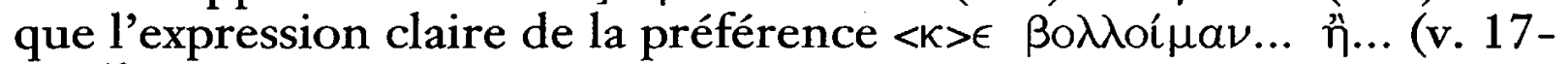
19) ${ }^{10}$.

De la première à la cinquième strophe, on passe de la multitude anonyme à la singularité nommée, mais les points de comparaison portent les deux fois sur le mouvement et l'éclat: puisqu'à la grâce de la marche ordonnée et rythmée d'une armée répond la démarche gracieuse de la femme aimée ${ }^{11}$. Or ces deux motifs sont aussi des éléments traditionnels de la description homérique des armées. C'est ainsi que l'Iliade présente l'armée des Achéens au chant XIX (v. 357362):

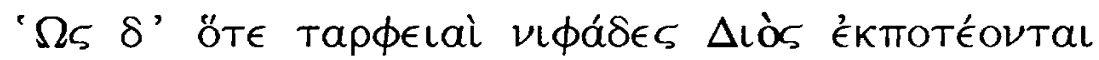

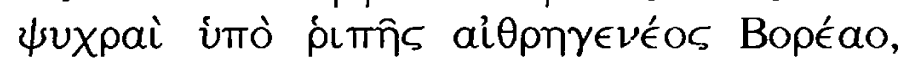

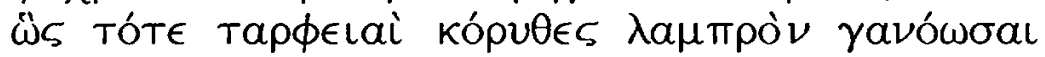

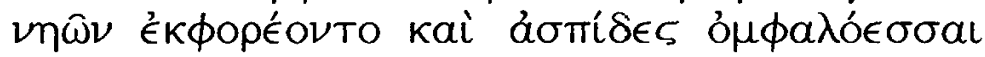

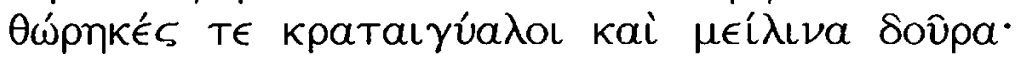

ä̌ $\gamma \lambda \eta \delta^{\prime}$ oủpavòv

Comme lorsque la neige de Zeus s'envole, tombant en flocons serrés, froide, sous le souffle impétueux de Borée issu de l'éther, de même alors, les casques à l'éclat resplendissant, serrés, sortaient des vaisseaux, et les boucliers bombés, les cuirasses solidement ajustées et les lances de frêne.

La lueur en atteignait le ciel [...].

La comparaison met en parallèle la chute des flocons de neige et la descente des guerriers qui sortent des vaisseaux. Sont mises en valeur les notions de multitude, de mouvement et d'éclat: multitude, avec la

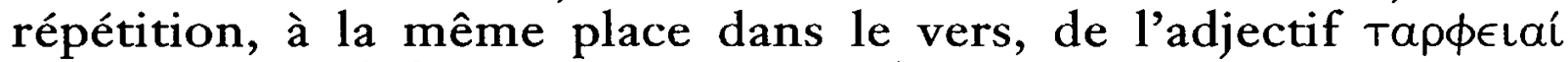
(v. 357, 359) et la longue énumération des vers 359-361; mouvement,

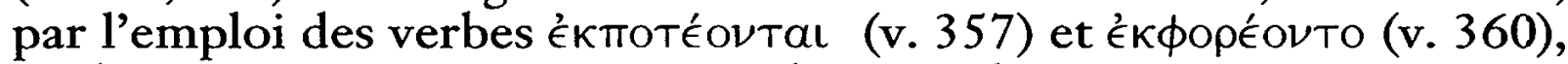
et du nom $\dot{\rho} \iota \pi \tilde{\varsigma}$ (v. 358); et éclat avec l'insistance marquée par

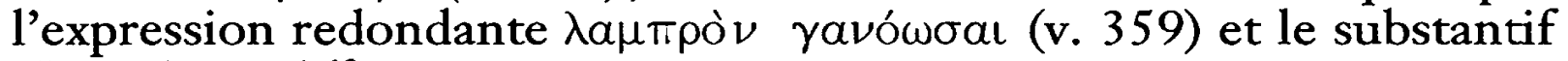
al $\gamma \lambda \eta$ (v. 362) ${ }^{12}$.

Il est donc particulièrement pertinent pour Sappho de souligner l'éclat qui caractérise Anactoria puisque c'est en cela même que la

10. Voir G. M. Kirkwood, Early Greek Monody, p. 107-108.

11. Voir A. P. Burnett, Three Archaic Poets, p. 280 ss.

12. Si l'éclat des guerriers homériques se donne à voir dans le but essentiel d'effrayrer l'ennemi, c'est-à-dire celui qui le voit, le thème de la vision est également présent chez Sappho, puisque la locutrice du poème 16 souhaite «voir $(t \delta \eta \nu)$ la démarche désirable et le rayonnant éclat du visage». Mais alors que l'effet du spectacle de l'éclat chez Homère est en général la panique (de l'ennemi), ce sont le désir et l'amour qui sont évoqués par la poétesse de Lesbos. 


\section{GAIA 10}

jeune femme est comparable et supérieure à la splendeur de l'armée lydienne. Mais on peut sans doute également penser que la poétesse rivalise ici aussi avec Homère : reprenant le thème de l'éclat,- associé par le poète épique au monde de la guerre -, pour en faire le point de comparaison entre une armée (précisément) et la beauté d'une jeune femme, et déplacer ainsi ce motif vers la sphère amoureuse, Sappho reconnaît certes une certaine allégeance au poète épique, mais, surtout, elle affirme avec force sa différence et ses choix.

L'éclat chez Homère n'est pas uniquement visuel, il peut aussi se faire sonore et en cela tout aussi remarquable. C'est ce qu'illustre ce passage du chant XVIII de l'Iliade (v. 205-206 et 217-221) décrivant l'apparition d'Achille sur le champ de bataille:

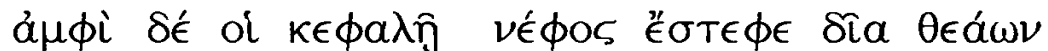

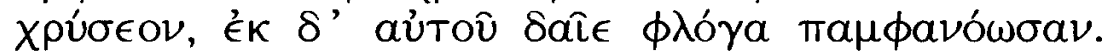

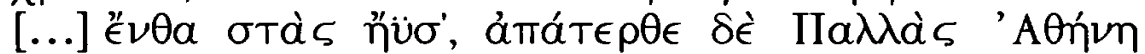

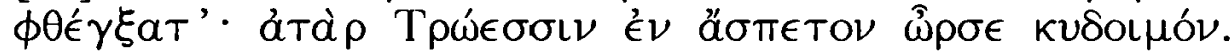

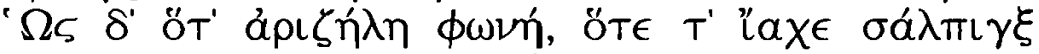

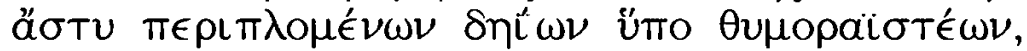

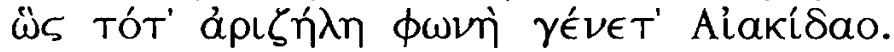

(Athéna) divine entre les déesses autour de sa tête répandit une nuée d'or, et de son corps fit jaillir une flamme resplendissante. [...]

Il s'arrêta (près du fossé) et poussa un cri, et Pallas Athéna, de son côté, fit entendre sa voix. Il suscita alors chez les Troyens un tumulte indicible.

On aurait dit la voix éclatante que fait entendre la trompette, le jour où des ennemis, destructeurs de vies humaines, entourent une cité; ainsi, éclatante, sonna la voix de l'Eacide.

Ce sont à la fois le spectacle de cet éclat extraordinaire qui émane d'Achille et le cri éclatant qu'il pousse à plusieurs reprises qui justi-

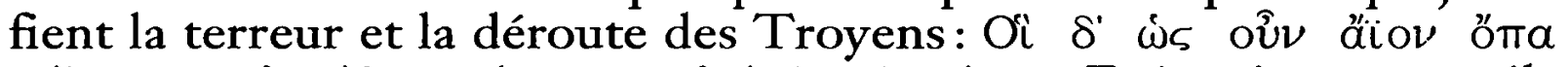

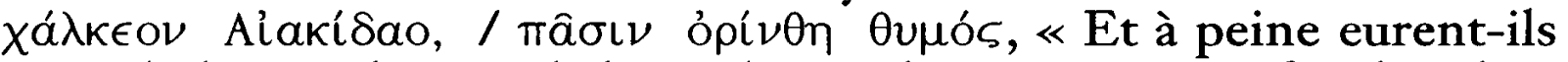
entendu la voix d'airain de l'Eacide, que leur cœur à tous fut ébranlé »

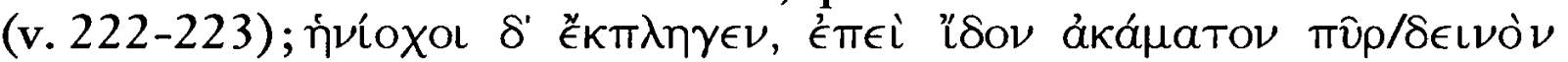

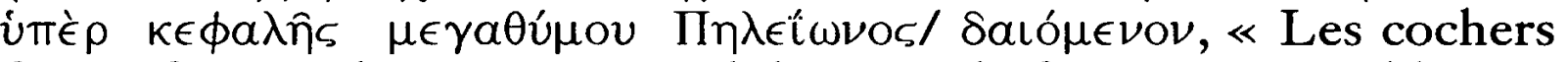
furent frappés de terreur, quand ils virent le feu vivace, terrible, qui flamboyait sur la tête du magnanime Péléide » (v. 225-227)...

Sappho semble adapter ce nouveau thème dans le très célèbre poème 31 :

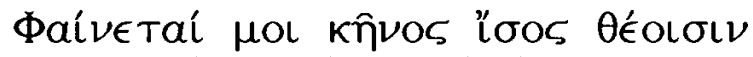

"̈ $\mu \mu \epsilon \nu$ '

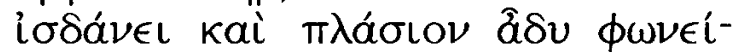

бas Ủmakoúel

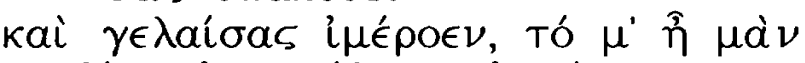

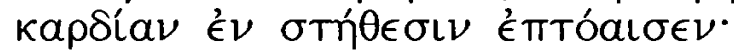




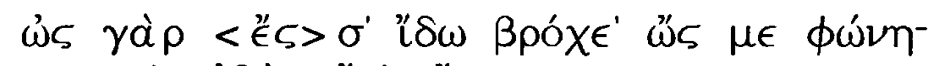

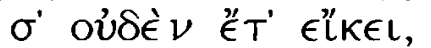

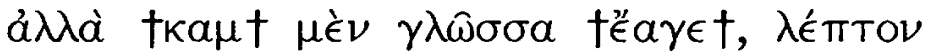

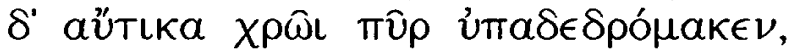

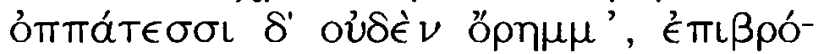

$\mu \epsilon \iota \sigma \mathrm{l} \delta^{\prime}$ ăkovaı,

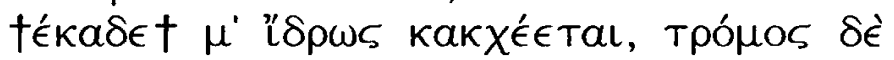

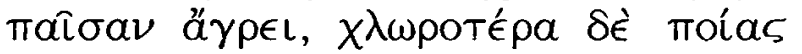

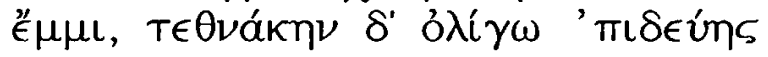

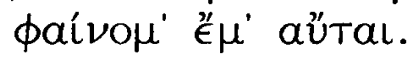

Il me semble être l'égal des dieux

l'homme qui en face de toi

est assis et tout près écoute

ta voix suave

et ton rire charmant qui, je le jure,

a frappé mon cour de passion dans ma poitrine;

car dès que je t'aperçois un instant, alors

il ne m'est plus possible d'articuler une parole,

mais ma langue se brise, et subtil

aussitôt sous ma peau court un feu;

dans mes yeux, aucun regard;

mes oreilles bourdonnent;

la sueur coule sur moi, un tremblement

me saisit toute; je suis plus verte que l'herbe;

et il me semble que pour un peu je suis morte.

Sappho décrit ici les symptômes de la passion. Cette passion s'empare d'elle quand elle voit la jeune fille qu'elle aime parler et rire en compagnie d'un homme. Elle brosse un portrait très détaillé des sensations physiques de son émotion en mêlant des éléments traditionnels, homériques, à des innovations personnelles. Ainsi, dans l'Iliade, quand Antiloque apprend la mort de Patrocle, il reste « longtemps sans pouvoir prononcer un mot, ses yeux se remplirent de

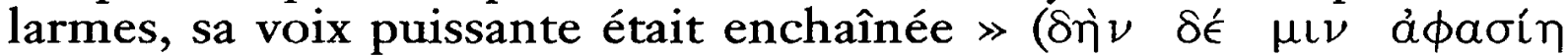

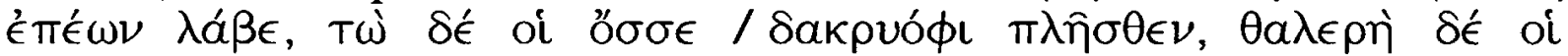
"E $\sigma \chi \in$ To $\phi \omega \nu \eta ́$, Iliade XVII, v. 695-696). Alexandre devant Ménélas est comme un homme qui a vu un serpent: « un frisson s'empare de ses membres, et il bat en retraite tandis que la pâleur envahit ses joues »

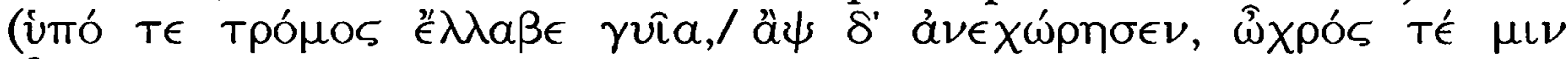

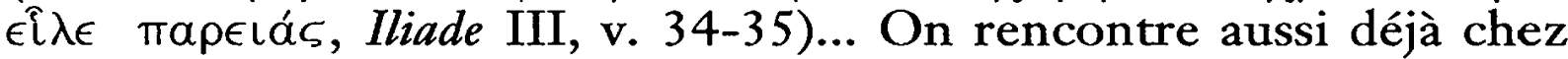
Homère ces autres symptômes que sont la cécité, la transpiration et jusqu'à l'évanouissement ${ }^{13}$. En revanche, la cause de ces symptômes

13. Pour la cécité, voir Iliade XV, v. 421 : Hector en est victime quand il voit son frère Polydore s'effondrer en tenant ses entrailles dans ses mains. Pour la transpiration, voir 
est différente: si, chez Homère, de telles manifestations physiques sont engendrées par la frayeur, le chagrin, la douleur, ce qui bouleverse au contraire Sappho dans le poème 31, ce sont la voix et le rire de la jeune fille. Or le rire est par nature éclat comme le suggèrent l'étymologie du verbe $\gamma \in \lambda a ́ \omega$, - rattachée à la même racine que

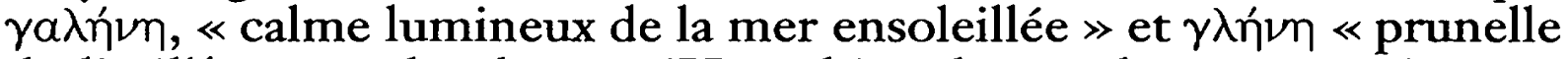
de l'œill ${ }^{14} \gg-$, et la glose qu'Hésychius donne de $\gamma \in \lambda \in \hat{\imath} \nu: \lambda a ́ \mu \pi \epsilon \iota \nu$. C'est aussi le sens que prend le verbe $\gamma \in \lambda \alpha \omega \omega$ quand il a la terre pour sujet; ainsi au moment où Achille revêt sa nouvelle armure au milieu des troupes achéennes, l'éclat resplendissant des casques, des boucliers, des cuirasses et des lances se propage à la fois verticalement et

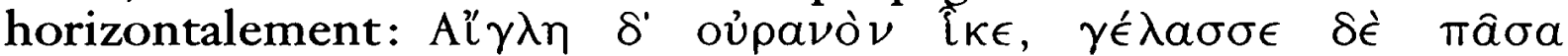

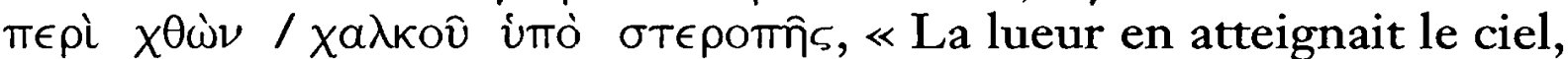
et la terre tout entière alentour se mit à resplendir sous l'éclair du bronze » (Iliade XIX, v. 362-363). Il semble donc qu'on puisse attribuer à cet éclat du rire, exprimé dans le poème de Sappho par le participe $\gamma \in \lambda a i ́ \sigma a \varsigma$, l'ensemble des symptômes physiques dont est victime la locutrice : l'éclat sonore a aussi un effet, et un effet désastreux sur celle qui le perçoit. En ce sens, l'amour ne serait donc pas si loin de la guerre.

On peut enfin remarquer que l'une des manifestations les plus frappantes (celle qui est le mieux mise en valeur par sa position finale dans l'énumération) est la pâleur livide, c'est-à-dire la perte de l'éclat

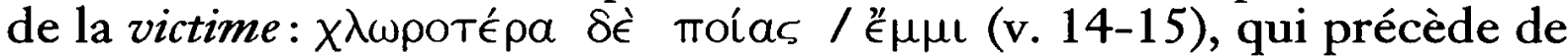

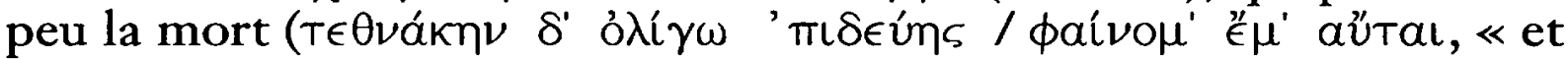
il me semble que pour un peu je suis morte $\gg, v$. 15-16). Sappho se livre donc ici encore à une adaptation multiple et originale des modèles homériques à sa propre poésie, personnelle et intime, pour décrire la passion amoureuse.

Chez Homère la brillance est par ailleurs souvent reconnue comme l'une des caractéristiques des corps célestes, soleil, astres, lune. On trouve la même représentation chez Sappho, à cette différence près que la lune semble l'emporter sur les autres astres comme l'illustre clairement le poème 34 :

Odyssée XX, v. 204 : la sueur s'empare du bouvier au moment où il aperçoit Ulysse. Pour la pâleur, voir Iliade X, v. 376 : Dolon est blême $(\chi \lambda \omega \rho o ́ s)$ de peur devant Diomède, il balbutie en outre et claque des dents. Pour l'évanouissement, voir Iliade XXII, v. 466 ss. : Andromaque défaille quand elle aperçoit le corps d'Hector traîné par Achille.

14. Voir P. Chantraine, Dictionnaire étymologique de la langue grecque. Histoire des mots, Paris, Klincksieck, 1968, s.v. 


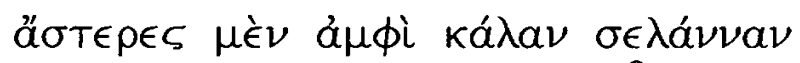

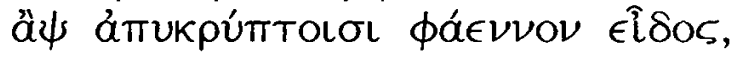

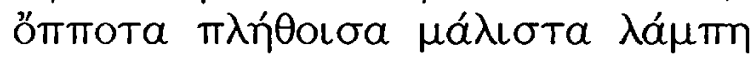
$\gamma \widehat{\alpha} \nu \ldots$

ả $\rho \gamma v p i ́ a$

Les étoiles autour de la belle lune de nouveau cachent leur brillant aspect, quand, dans sa plénitude, elle resplendit de son plus vif éclat sur la terre...

$$
\text { d'argent }
$$

Ces vers ne peuvent manquer de rappeler certains passages homériques, comme cet extrait du chant VIII de l'Iliade (v. 555-556):

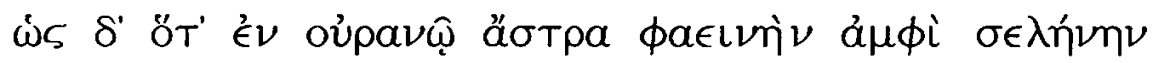

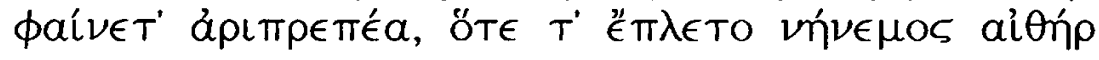

Ainsi luisaient les étoiles dans le ciel, autour de la brillante lune, éclatantes, les jours où l'éther était sans vent.

Mais, tandis que la lune de l'Iliade semble dotée d'une brillance égale à celle des étoiles, celle de Sappho l'emporte au contraire nettement sur les autres astres. Il est vraisemblable que le fragment 34 de la poétesse soit la première partie $(\mu \epsilon \epsilon)$ d'une comparaison, dans laquelle Sappho évoquait l'une de ses compagnes qui surpassait en beauté toutes les autres. L'ensemble des commentateurs s'accordent ainsi pour considérer que l'éclat de la nature sert sans doute ici d'analogie pour la beauté. Cette interprétation est en partie soutenue par le parallèle avec le poème 96 , qui met explicitement en scène cette comparaison:

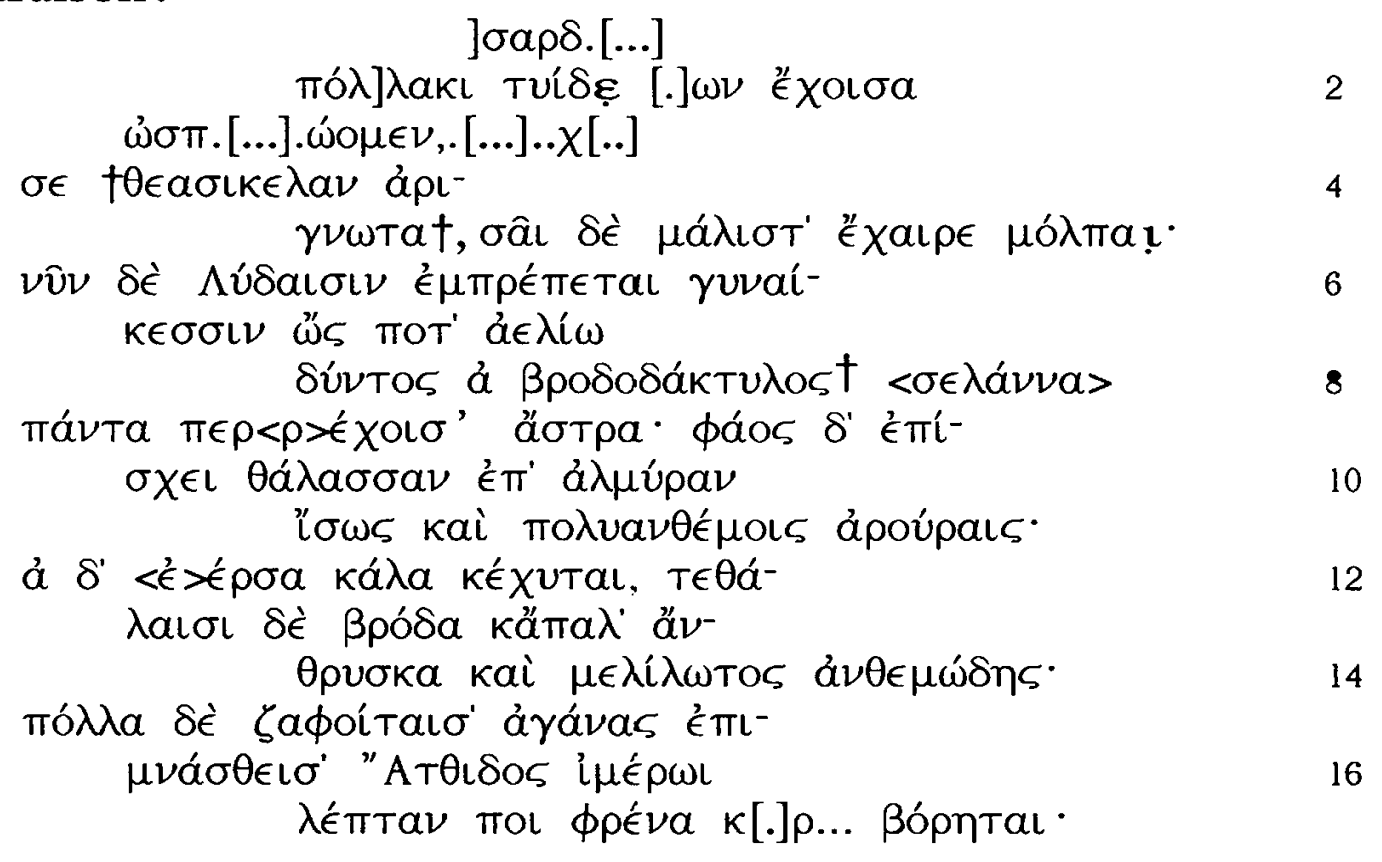




\section{GAIA 10}

[...] à Sardes... (?)

Souvent je pense à elle, (comme nous vivions?).

Toi semblable à une déesse, facile à reconnaître, et, par-dessus tout, elle se réjouissait de ton chant. Mais maintenant, parmi les femmes lydiennes, elle se distingue comme, une fois le soleil couché, la lune aux doigts de rose,

l'emportant sur toutes les étoiles; et sa clarté s'étend sur la mer salée comme aussi sur les champs aux multiples fleurs; et la rosée s'épand en beauté, et s'épanouissent les roses, le tendre cerfeuil et le mélilot en fleurs. Souvent elle va et vient, se souvenant de l'aimable Atthis; le désir oppresse son âme délicate.

La lune constitue ici clairement un symbole de la beauté de la femme, même si elle ne tarde pas à s'affranchir du cadre de la comparaison pour prendre d'une certaine façon son indépendance, redevenir lune réelle et donner lieu à une description naturelle pleine de charme. On retrouve, par ailleurs, dans ce poème la même prééminence de la lune sur toutes les étoiles, qui illustre le contraste d'une lueur éclatante par rapport à d'autres, nombreuses, plus pâles. C'est pourquoi, commente Denys Page, la jeune fille absente est comparée non au soleil, mais à la lune qui surpasse en éclat d'autres sources de lumière brillante ${ }^{15}$.

Mais un problème au moins demeure, c'est celui que pose l'interprétation de l'épithète $\beta p \circ \delta \circ \delta a ́ k T u \lambda o \varsigma, ~ «$ aux doigts de rose », qualifiant la lune ${ }^{16}$. Certes, elle annonce la description du paysage qui suit

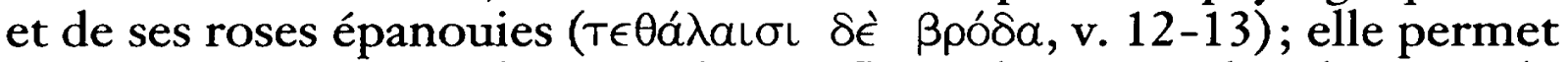
aussi une évocation discrète de ces fleurs liées à Aphrodite et à la sphère de l'amour. Mais, à l'évidence, cet adjectif ne renvoie pas dans le poème de Sappho à une réalité comparable à celle qu'exprime le

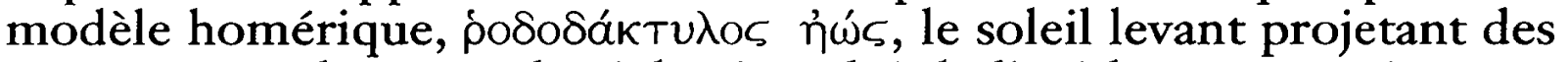
rayons rosés dans tout le ciel. L'emploi de l'épithète $\beta p o \delta o \delta a ́ k T U \lambda o \varsigma$ obéit donc à d'autres motivations. Sappho ne se serait-elle pas livrée à un jeu de variation sur la langue épique en transférant un qualificatif d'un nom à un autre? L'expression homérique étant célèbre, le vers

15. Voir D. L. Page, Sappho and Alcaeus, p. 95.

16. Voir en particulier D. L. Page, Sappho and Alcaeus, p. 90 ss. ; C. M. Bowra, Greek Lyric Poetry, p. 195 ; G. M. Kirkwood, Early Greek Monody, notes 33, p. 253-255 et 49, p. 261 ; E. Irwin, «The Crocus and the Kose...», p. 165-168 ; D.E. Gerber, A Companion to the Greek Lyric Poets, p. 180-181. 
de Sappho met en parallèle, de façon indirecte mais indubitable, la lune, clarté nocturne, et l'aurore, première lueur du matin ${ }^{17}$.

Or, Homère associe au contraire le soleil à l'aurore : Zeus a donné à Trôs, le père de Ganymède, en rançon de son fils, des chevaux qui « sont les meilleurs chevaux qui soient sous l'aurore et le soleil »

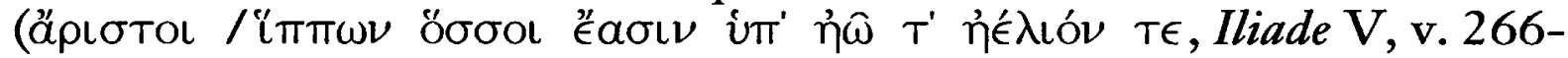
267); et Hector qui refuse d'ajouter foi aux présages des oiseaux déclare: « Ils peuvent bien aller à droite, vers l'aurore et le soleil,

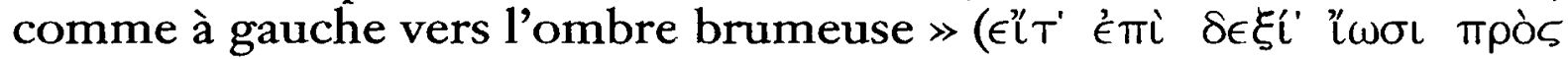

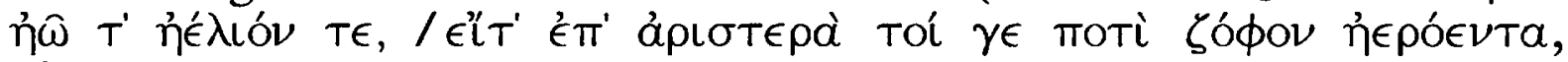
Iliade XII, v. 239-240).

Sappho opère donc bien un renversement du schéma homérique

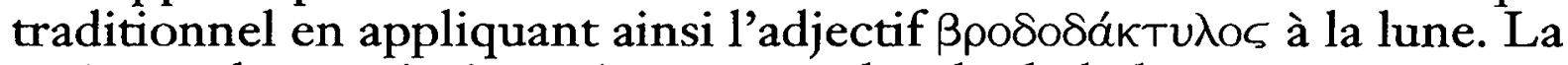
poétesse donne ainsi une image paradoxale de la lumière, suggérant un éclat exceptionnel de l'astre nocturne, dans un monde peut-être inversé, où les femmes ont la prééminence. On peut en effet relever plusieurs autres associations des femmes à l'astre nocturne, comme c'est le cas par exemple dans le fragment 154:

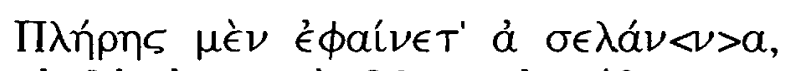

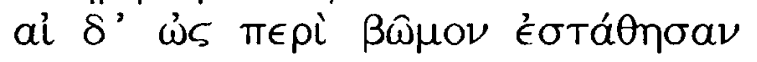

Pleine apparaissait la lune et quand elles se placèrent autour de l'autel...

Malheureusement le caractère lacunaire de ce poème et le fait que l'on ne sache rien de son contexte ne permettent pas de pousser plus loin l'analyse. Mais on retrouve l'union de la lune et de la femme dans un autre poème, plus explicite, le fragment $168 \mathrm{~B}^{18}$ :

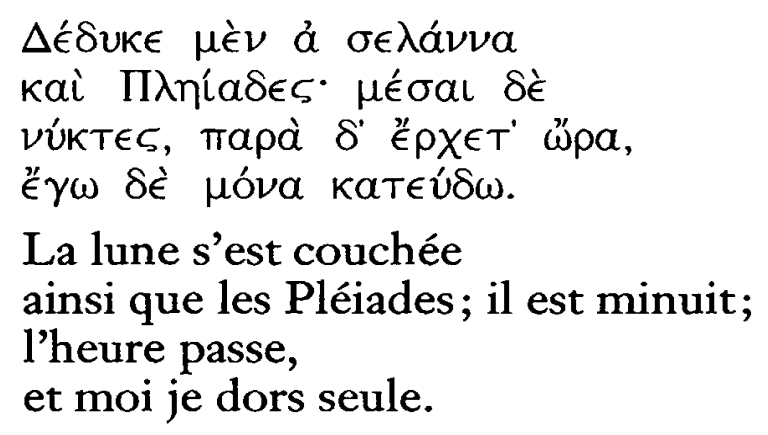

La lune s'est couchée

ainsi que les Pléiades; il est minuit;

l'heure passe,

et moi je dors seule.

17. On peut noter que Sappho attribue ailleurs à l'aurore un qualificatif proche de

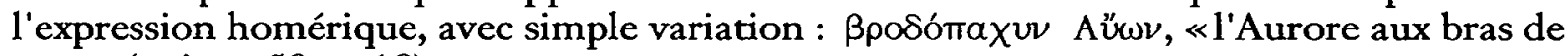
rose» (poème 58, v. 19).

18. L'attribution de ce fragment à Sappho est discutée : récusée par E. Lobel et D.L.Page (Poetarum Lesbiorum Fragmenta), elle est au contraire soutenue par E.M. Voigt (Sappho et Alcaeus). 
Ce texte présente une scène nocturne, qui suggère et souligne la solitude de la locutrice. On peut noter que tous les personnages ou éléments qui composent cette scène sont féminins. Le poème repose sur des effets de contraste et de parallélisme: l'association LunePléiades (ả $\sigma \in \lambda a ́ v \nu \alpha$ кai $\Pi \lambda \eta i ́ a \delta \epsilon \zeta)$ s'oppose au singulier nettement affirmé de la femme ( encore renforcée par le fait que non seulement le nom des astres mais encore celui de la nuit sont exprimés au pluriel: $\Pi \lambda \eta \operatorname{li}_{\alpha \delta} \delta 5, v u ́ k T \epsilon \zeta$. L'ensemble de la scène suggère une nette association des femmes avec le monde de la nuit, et en particulier avec la lune ${ }^{19}$.

Au terme de cette étude, nous pouvons donc affirmer que la poésie de Sappho joue délibérément avec le modèle homérique: en déclinant le motif de la brillance, la poétesse de Lesbos n'hésite pas à reprendre un thème épique connu pour exalter des vertus purement héroïques et masculines; mais elle le détourne de ce contexte guerrier pour l'adapter à celui de l'amour, de la beauté et de la femme. Loin de se laisser aveugler par l'éclat d'Homère, Sappho parvient, en maniant à la fois les images et les épithètes homériques d'une nouvelle façon, à créer sa propre poésie, originale et féminine.

\section{Bibliographie sommaire}

Bonnafé A., Poésie, nature et sacré. Tome I: Homère, Hésiode et le senti ment grec de la nature. Tome II: L'âge archaïque, Lyon, Maison de l'Orient Méditerranéen, I : 1984; II : 1987.

Bowra C.M., Greek Lyric Poetry from Alcman to Simonides, Oxford, Clarendon Press, 1961 ( $2^{\mathrm{e}}$ édition).

Burnett A.P., Three Archaic Poets. Archilochus, Alcaeus, Sappho, Londres, Duckworth, 1983.

Campbell D.A., Greek Lyric Poetry. A Selection of Early Greek Lyric, Elegiac and Iambic Poetry, Bristol, Bristol Classical Press, 1967, 1982.

—, Greek Lyric. I: Sappho and Alcaeus, Cambridge, Mass., Harvard University Press; Londres, W. Heinemann, 1982, Loeb Classical Library, réédité avec corrections, 1990.

19. On peut peut-être voir un autre parallélisme dans les actions marquées par les

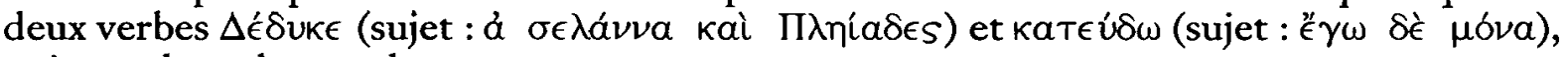
qui encadrent la strophe. 
-, The Golden Lyre. The Themes of the Greek Lyric Poets, Londres, Duckworth, 1983.

Duchemin J., Pindare, poète et prophète (chapitre "La mystique de l'or, de la lumière et des couleurs"), Paris, Belles Lettres, 1955, p. 191228.

Gerber D.E., A Companion to the Greek Lyric Poets, Leiden, Brill, 1997.

Harvey A.E., « Homeric Epithets in Greek Lyric Poetry », Classical Quarterly, 7, 1957, p. 206-223.

Irwin E., «The Crocus and the Rose : a Study of the Interrelationship between the Natural and the Divine World in Early Greek Poetry », dans Gerber D.E. ed., Greek Poetry and Philosophy. Studies in Honour of Leonard Woodbury, Chico (California), Scholars Press, 1984, p. 147-168.

Kirkwood G.M., Early Greek Monody. The History of a Poetic Type, Ithaca ; Londres, Cornell University Press, 1974.

Lasserre F., Sappho, une autre lecture, Padoue, ed. Antenore, 1989.

Lobel E. et Page D.L., Poetarum Lesbiorum Fragmenta, Oxford, Clarendon Press, 1955.

Mazon P., Homère. Iliade, Paris, Belles Lettres, CUF, 1987 ( $1^{\text {re }}$ édition 1937).

Page D.L., Sappho and Alcaeus. An Introduction to the Study of Ancient Lesbian Poetry, Oxford, Clarendon Press, 1955.

Pigeaud J., Sappho. Poèmes, Rivages poche, Petite Bibliotbèque, Paris, Éditions Payot \& Rivages, 2004.

Puech A. et Reinach T., Alcée, Sappho, Paris, Belles Lettres, CUF, 1960.

Rissman L., Love as war. Homeric Allusion in the Poetry of Sappho, Königstein, A. Hain, 1983. 